\title{
A Closed-Form Solution for the Distribution of the Sum of Nakagami- $m$ Random Phase Vectors
}

\author{
George K. Karagiannidis, Senior Member, IEEE
}

\begin{abstract}
The distribution of the modulus of the sum of random phase vectors, is of great practical importance in several applications which deal with sums of sinusoidal signals (wireless multipath transmissions, radars, optical communications, etc). In this letter, simple closed-form expressions are presented for the probability density function (PDF), the cumulative distribution function (CDF), the moment generating function (MGF) and the moments of the envelope distribution of the sum of $L$ nonidentical random Nakagami- $m$ phase vectors with integer fading parameters. Moreover, the average over this distribution of the Gaussian $Q$-function and of the squared $Q$-function, are also presented in closed-form.
\end{abstract}

Index Terms-Nakagami- $m$ fading channels, sum of random phase vectors, selective fading, Gaussian $Q$-function, OFDM, coherent modulation.

\section{INTRODUCTION}

$\mathbf{T}$ HE statistics of the sum of several random sinusoidal signals -which can be considered as sums of random vectors $(\mathrm{RVs})$ and is usually referred in the literature as the random vector problem- is encountered in several applications, including communications over multipath fading channels, radars, light scattering, etc.

Recent publications related to the problem of RVs include [1] and [2]. In the pioneering work of Abdi et. al. [1], a general approach was presented for the evaluation of the probability density function (PDF) of the sum of an arbitrary number of RVs with arbitrary statistics. Moreover, this paper includes a complete literature review, concerning this problem. Recently, Du et. al. [2], proved a result for the PDF of the sum of Nakagami- $m$ RVs, which was stated without proof many years ago by Nakagami in [3] and is a special case of [1]. Moreover, in the same work it was shown that a further approximation made by Nakagami, although it is a useful tool for rapid analysis, can however be inaccurate. Next, a Nakagami- $m$ phase vector is considered to have its modulus following Nakagami- $m$ distribution [3], while its phase is uniformly distributed.

In this letter, closed-form expressions, in terms of elementary functions, are derived for the PDF, the cumulative distribution function (CDF), the moment generating function (MGF) and the moments of the envelope distribution of a sum of $L$ non-identical Nakagami- $m$ random phase vectors with integer fading parameters. Moreover, closed-form formulae are

Manuscript submitted June 28, 2006. The associate editor coordinating the review of this letter and approving it for publication was Dr. Mischa Dohler.

G. K. Karagiannidis is with the Department of Electrical and Computer Engineering, Aristotle University of Thessaloniki, 54124 Thessaloniki, Greece (e-mail: geokarag@auth.gr).

Digital Object Identifier 10.1109/LCOMM.2006.060991. derived for the average over this distribution of the Gaussian $Q$-function and of the squared $Q$-function, which can be efficiently used to evaluate the error performance of several coherent modulations over frequency-selective Nakagami- $m$ fading channels.

\section{The Distribution of the Modulus of the Sum of $L$ NAKAGAMI- $m$ RANDOM PHASE VECTORS}

Consider $L$ phase vectors, $h_{i}, i=1, \ldots, L$, with amplitudes (modulus) $\left|h_{i}\right|$ and phases $\phi_{i}$, where $\left|h_{i}\right|$ follows Nakagami-m distribution with PDF [3]

$$
f_{\left|h_{i}\right|}(r)=\frac{2 m_{i}^{m_{i}}}{\Omega_{i}^{m_{i}} \Gamma\left(m_{i}\right)} r^{2 m_{i}-1} \exp \left(-\frac{m_{i}}{\Omega_{i}} r^{2}\right)
$$

where $\Gamma(x)$ is the Gamma function [4, (8.310/1)], $\Omega_{i}=$ $\mathcal{E}\left\langle\left|h_{i}\right|^{2}\right\rangle, m_{i}=\Omega_{i}^{2} / \mathcal{E}\left\langle\left(\left|h_{i}\right|^{2}-\Omega\right)^{2}\right\rangle \geq 1 / 2$, and $\mathcal{E}\langle\cdot\rangle$ denotes expectation. The PDF in (1) includes the cases of Rayleigh $(m=1)$ and one-sided Gaussian $(m=1 / 2)$ distributions as special ones. Furthermore, it is assumed that the phases $\phi_{i} \mathrm{~s}$ are mutually independent and independent of the amplitudes $\left|h_{i}\right| \mathrm{s}$, as it is satisfied in the most practical cases [1]. The problem is to obtain the distribution of the amplitude of the vector

$$
H=\sum_{i=1}^{L}\left|h_{i}\right| e^{j \phi_{i}} .
$$

In [3], Nakagami stated without proof that a solution to this problem is the integral representation

$$
f_{|H|}(r)=r \int_{0}^{\infty} \prod_{i=1}^{L}{ }_{1} F_{1}\left(m_{i} ; 1 ;-\frac{\Omega_{i}}{4 m_{i}} x^{2}\right) J_{0}(r x) x d x
$$

with ${ }_{1} F_{1}(a ; b ; z)$ being the confluent hypergeometric function and $J_{0}(z)$ the zeroth-order Bessel function [4]. A proof to (3) only recently was given in [2].

Although the integral representation in (3) -as it was shown by simulations in [2]- is exact, a numerical evaluation of this formula seems to be difficult for $L \geq 3$, since the product $\prod_{i=1}^{L}{ }_{1} F_{1}\left(m_{i} ; 1 ;-\frac{\Omega_{i}}{4 m_{i}} x^{2}\right)$ rapidly decreases with an increase of $\frac{\Omega_{i}}{4 m_{i}} x^{2}$ and the multiplication of several small numbers could lead to inaccurate results, when numerical integration is performed. The difficulty to evaluate numerically the integral in (3) can be also verified when using MATHEMATICA software with $L=4, m_{i}=m=3, \Omega_{i}=\Omega=15 \mathrm{~dB}$ and $r=5$. In this case, the CPU time needed is $125 \mathrm{sec}$, with the program being unable to check the accuracy of the convergence. 
Next, simple closed-form expressions for the PDF, the CDF and the MGF of $|H|$ are presented, when $m_{i}$ takes integer values. We first define the operator $\mathcal{G}\langle$.$\rangle as$

$\mathcal{G}\langle\mathcal{X}\rangle \triangleq \sum_{i_{1}=0}^{m_{1}-1} \cdots \sum_{i_{L}=0}^{m_{L}-1} \sum_{j=0}^{S_{L}} \frac{\prod_{k=1}^{L}\left(\frac{\left(1-m_{k}\right)_{i_{k}}}{\left(i_{k} !\right)^{2}}\right)\left(-S_{L}\right)_{j} S_{L} ! Y_{L} \mathcal{X}}{(j !)^{2} U_{L}^{S_{L}}}$

where $\mathcal{X}$ is a mathematical expression and $\mathcal{G}\langle\mathcal{X}\rangle$ means replacement of the $\mathcal{X}$ in the second part of (4) with this expression. Moreover, $S_{L}=\sum_{k=1}^{L} i_{k}, Y_{L}=\prod_{k=1}^{L}\left(\frac{\Omega_{k}}{4 m_{k}}\right)^{i_{k}}$, $U_{L}=\sum_{k=1}^{L} \frac{\Omega_{k}}{4 m_{k}}$ and $(z)_{n}$ is the Pochhammer symbol [5, (6.1.22)] with $(-z)_{n}=(-1)^{n}(z-n+1)_{n}$. Note, that $\mathcal{G}\langle\mathcal{X}\rangle$ has useful properties such as

$$
\int_{a}^{b} \mathcal{G}\langle\mathcal{X}\rangle d x=\mathcal{G}\left\langle\int_{a}^{b} \mathcal{X} d x\right\rangle
$$

which will be used below.

\section{A. $P D F$}

Using (5) and $[4,(8.972 .1)$ and (8.970.1)] the PDF of $|H|$ can be written as

$$
\begin{aligned}
f_{|H|}(r) & =\sum_{i_{1}=0}^{m_{1}-1} \cdots \sum_{i_{L}=0}^{m_{L}-1} \prod_{k=1}^{L}\left(\frac{\left(1-m_{k}\right)_{i_{k}}}{\left(i_{k} !\right)^{2}}\right) r \\
& \times \int_{0}^{\infty} e^{-U_{L} x^{2}} x^{2 S_{L}+1} J_{0}(x r) d x .
\end{aligned}
$$

The integral in (6) can be solved in closed-form using [4, (6.631.1)], yielding to

$$
\begin{aligned}
f_{|H|}(r) & =\sum_{i_{1}=0}^{m_{1}-1} \cdots \sum_{i_{L}=0}^{m_{L}-1} \prod_{k=1}^{L}\left(\frac{\left(1-m_{k}\right)_{i_{k}}}{\left(i_{k} !\right)^{2}}\right) r \\
& \times\left(\frac{\Gamma\left(S_{L}+1\right)}{2 U_{L}^{S_{L}+1}}{ }_{1} F_{1}\left(S_{L}+1 ; 1 ;-\frac{r^{2}}{4 U_{L}}\right)\right) .
\end{aligned}
$$

After the replacement of the confluent hypergeometric functions with their finite series representations $[4,(8.972 .1)$ and (8.970.1)], $f_{|H|}(r)$ can be finally written as a finite sum of elementary functions

$$
f_{|H|}(r)=\mathcal{G}\left\langle\frac{e^{-\frac{r^{2}}{4 U_{L}}} r^{2 j+1}}{U_{L}^{j+1} 2^{2 j+1}}\right\rangle
$$

which is in fact a sum of weighted Nakagami- $m$ PDFs.

\section{B. $C D F$}

The CDF of $|H|$ is defined as

$$
F_{|H|}(R)=\int_{0}^{R} f_{|H|}(r) d r
$$

or

$$
\begin{aligned}
F_{|H|}(R) & =\int_{0}^{R} \mathcal{G}\left\langle\frac{e^{-\frac{r^{2}}{4 U_{L}}} r^{2 j+1}}{U_{L}^{j+1} 2^{2 j+1}}\right\rangle d r \\
& =\mathcal{G}\left\langle\frac{1}{U_{L}^{j+1} 2^{2 j+1}} \int_{0}^{R} e^{-\frac{r^{2}}{4 U_{L}}} r^{2 j+1} d r\right\rangle \\
& =\mathcal{G}\left\langle j !-\Gamma\left(j+1, \frac{R^{2}}{4 U_{L}}\right)\right\rangle \\
& =\mathcal{G}\left\langle j !\left(1-e^{-\frac{R^{2}}{4 U_{L}}} \sum_{n=0}^{j} \frac{R^{2 n}}{2^{2 n} U_{L}^{n} n !}\right)\right\rangle
\end{aligned}
$$

where in (10), [5, (3.381.1)] is used to solve the integral and $[4,(8.352 .2)]$ to replace the incomplete Gamma function $[4$, (8.350.2)], $\Gamma(x, y)$, with its finite series representation.

\section{C. $M G F$}

The MGF of $|H|$ is defined as

$$
M_{|H|}(s)=\int_{0}^{\infty} e^{s r} f_{|H|}(r) d r
$$

or using [4, (3.462.1) and (9.240)]

$$
\begin{aligned}
M_{|H|}(s) & =\int_{0}^{\infty} \mathcal{G}\left\langle\frac{e^{-\frac{r^{2}}{4 U_{L}}+s r} r^{2 j+1}}{U_{L}^{j+1} 2^{2 j+1}}\right\rangle d r \\
& =\mathcal{G}\left\langle\frac{1}{U_{L}^{j+1} 2^{2 j+1}} \int_{0}^{\infty} e^{-\frac{r^{2}}{4 U_{L}}+s r} r^{2 j+1} d r\right\rangle \\
& =\mathcal{G}\left\langle j !_{1} F_{1}\left(j+1 ; \frac{1}{2} ; U_{L} s^{2}\right)\right. \\
& \left.+2 s \Gamma\left(\frac{3}{2}+j\right) \sqrt{U_{L}} F_{1}\left(j+\frac{3}{2} ; \frac{3}{2} ; U_{L} s^{2}\right)\right\rangle .
\end{aligned}
$$

The above expression for the MGF of $|H|$ can be efficiently utilize the well-known MGF approach [6], to evaluate the error performance of orthogonal frequency-division multiplexing (OFDM) communications in Nakagami- $m$ frequency selective fading channels [2].

\section{Moments}

The $N$ th moment of $|H|$ can be written as

$$
\begin{aligned}
\mu_{N} & =\int_{0}^{\infty} \mathcal{G}\left\langle\frac{e^{-\frac{r^{2}}{4 U_{L}}} r^{2 j+1+N}}{U_{L}^{j+1} 2^{2 j+1}}\right\rangle d r \\
& =\mathcal{G}\left\langle\frac{1}{U_{L}^{j+1} 2^{2 j+1}} \int_{0}^{\infty} e^{-\frac{r^{2}}{4 U_{L}}} r^{2 j+1+N} d r\right\rangle \\
& =\mathcal{G}\left\langle 2^{N} U_{L}^{\frac{N}{2}} \Gamma\left(\frac{N}{2}+j+1\right)\right\rangle
\end{aligned}
$$

where $[5,(3.381 .3)]$ is used to solve the integral in (13). The most important first (mean) and second (variance) moment, can be easily computed from (13), using $N=1$ and $N=2$, respectively. 


\section{The Average OVER $|H|$ OF THE GAUSSIAN $Q$-FUNCTION}

When characterizing the performance of coherent digital communications, the generic form of the expression for the error probability involves the Gaussian $Q$-function (or for quadrature amplitude modulation (QAM) the square of the Gaussian $Q$-function) with an argument proportional to the instantaneous signal-to-noise ratio (SNR) of the received signal. To compute the average error probability in theses cases, one must evaluate the average over the fading PDF of the $Q$-function or the squared $Q$-function [6].

\section{A. The average of $Q(A r)$}

The average over $|H|$ of the Gaussian $Q$-function is by definition

$$
\mathcal{E}\langle Q(A r)\rangle=\int_{0}^{\infty} Q(A r) f_{|R|}(r) d r
$$

where $A$ is a factor related to SNR. With the help of (4), (14) can be written as

$$
\begin{aligned}
\mathcal{E}\langle Q(A r)\rangle & =\int_{0}^{\infty} \mathcal{G}\left\langle\frac{e^{-\frac{r^{2}}{4 U_{L}}} r^{2 j+1}}{U_{L}^{j+1} 2^{2 j+1}} Q(A r)\right\rangle d r \\
& =\mathcal{G}\left\langle\frac{1}{U_{L}^{j+1} 2^{2 j+1}} \int_{0}^{\infty} e^{-\frac{r^{2}}{4 U_{L}}} r^{2 j+1} Q(A r) d r\right\rangle .
\end{aligned}
$$

Fortunately, the integral in (15) is encountered in the average over Nakagami- $m$ fading channels of the $Q$-function, and can be solved in terms of elementary functions using [6, (5.17)], finally resulting in

$$
\mathcal{E}\langle Q(A r)\rangle=\mathcal{G}\left\langle\frac{j !}{2}-\frac{j ! s}{2} \sum_{n=0}^{j}\left(\begin{array}{c}
2 n \\
n
\end{array}\right)\left(\frac{1-s^{2}}{4}\right)^{n}\right\rangle
$$

where $c=2 A^{2} U_{L}$ and $s=\sqrt{\frac{c}{1+c}}$. Note, that for the case of arbitrary values of $m_{i}$, a solution for $\mathcal{E}\langle Q(A r)\rangle$ was given in [2], in terms of the Lauricella function.

\section{B. The average of $Q^{2}(A r)$}

Following the same procedure as below, the average over $|H|$ of the squared Gaussian $Q$-function can be written as

$$
\mathcal{E}\langle Q(A r)\rangle=\mathcal{G}\left\langle\frac{1}{U_{L}^{j+1} 2^{2 j+1}} \int_{0}^{\infty} e^{-\frac{r^{2}}{4 U_{L}}} r^{2 j+1} Q^{2}(A r) d r\right\rangle
$$

where the integral in (17) is also encountered in the average over Nakagami- $m$ fading channels of the squared of $Q$ function and can be also evaluated in closed-form using [6, (5.30)].

Finally (17) yields

$\mathcal{E}\left\langle Q^{2}(A r)\right\rangle=$

$\mathcal{G}\left\langle\frac{j !}{4}-\frac{j !}{\pi} s\left(\left(\frac{\pi}{2}-\tan ^{-1} s\right) \sum_{n=0}^{j} \frac{\left(\begin{array}{c}2 n \\ n\end{array}\right)}{(4+4 c)^{n}}-\right.\right.$

$$
\left.\left.\sin \left(\tan ^{-1} s\right) \sum_{n=1}^{j} \sum_{t=1}^{n} \frac{\left(\begin{array}{c}
2 n \\
n
\end{array}\right)\left[\cos \left(\tan ^{-1} s\right)\right]^{2(n-t)+1}}{\left(\begin{array}{c}
2(n-t) \\
n-t
\end{array}\right) 4^{t}[2(n-t)+1]}\right)\right\rangle .
$$

\section{DISCUSSION}

a) The simple closed-form expressions, presented above, can be efficiently used to study the error performance of several digital modulations (including square QAM), in the case where the fading environment can be modelled as a sum of not necessarily identical random Nakagami- $m$ phase vectors (e.g. OFDM in frequency-selective fading channels [2]).

b) Due to the form of the operator $\mathcal{G}\langle$.$\rangle , the approach$ presented in this letter can be easily extended to the case where diversity is used at the receiver (e.g maximal ratio combining (MRC) or selection combining (SC)).

c) Following the same procedure, closed-form expressions can be also derived for other important utilities, as the average of the $\log \left(1+r^{2}\right)$ over $|H|$, which is related to the average Shannon capacity.

\section{REFERENCES}

[1] A. Abdi, H. Hashemi, and S. Nader-Efstahani, "On the pdf of the sum of random vectors," IEEE Trans. Commun., vol. 48, no. 1, pp. 7-12, Jan. 2000.

[2] Z. Du, J. Cheng, and N. C. Beaulieu, "Accurate error-rate performance analysis of OFDM on frequency-selective nakagami- $m$ fading channels," IEEE Trans. Commun., vol. 54, no. 2, pp. 319-328, Feb. 2006.

[3] M. Nakagami, "The $m$-distribution - A general formula of intensity distribution of rapid fading," Statistical Methods in Radio Wave Propagation, vol. 40, pp. 757-768, Nov. 1962.

[4] I. S. Gradshteyn and I. M. Ryzhik, Table of Integrals, Series, and Products, Fifth Edition. New York: Academic, 1994.

[5] M. Abramovitz and I. A. Stegun, Handbook of Mathematical Functions with Formulas, Graphs, and Mathematical Tables, Ninth Edition. New York: Dover, 1972.

[6] M. K. Simon and M.-S. Alouini, Digital Communication over Fading Channels, Second Edition. New York: Wiley, 2005. 